\title{
DNGK - Forschung in Zeiten von COVID-19
}

\author{
Die COVID-19-Pandemie stellt viele Nachwuchsforscher vor bisher ungeahnte Herausforde-
} rungen. Die Erreichbarkeit vulnerabler Gruppen sowie die Frage nach der Repräsentativität der eigenen Datensätze müssen häufig vor neuen Bedingungen verhandelt werden. Anfang März veranstaltete die DNGK-Arbeitsgruppe Nachwuchsförderung ein öffentliches Webseminar, das damit verbundene Herausforderungen und mögliche Lösungsansätze beleuchtete. Das Seminar fand im Rahmen der DNGK-Webseminarreihe statt.

Lisa Bach, Karin Drixler, Janine Michele, Jacqueline Posselt

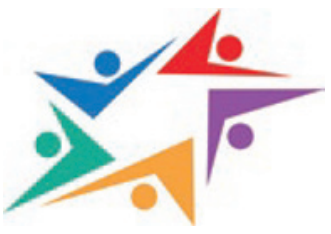

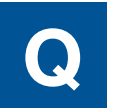

ualitative Forschungsmethoden sind häufig von persönlichem Kontakt gekennzeichnet, beispielsweise innerhalb der Fokusgruppen und bei Interviews. Kontaktreduktionen zur Eindämmung der Pandemie haben somit direkte Auswirkungen auf die gängigen Forschungsmethoden.

Qualitative Forschung müsse jedoch nicht unbedingt face-to-face erfolgen, betonte Saskia Jünger, Professorin für Forschungsmethoden im Kontext Gesundheit an der Hochschule für Gesundheit in Bochum, im Rahmen des DNGK-Webseminars der Arbeitsgruppe Nachwuchsforschung. Es sei wichtig, den digitalen Raum zu erkunden und weitere Formen der Datenerhebung in den Blick zu nehmen. Auch die digitale Landschaft sei real, insbesondere in der qualitativen Forschung suche man deshalb Antworten auf die Frage nach guter digitaler Kommunikation. „Forschende sollten auch den digitalen Raum als ethnographisches Forschungsfeld betrachten und dabei die eigene Rolle sowie die neuen Gegebenheiten reflektieren“, erklärte Jünger. Zudem müsse man herausfinden, wie sich Kommunikationsformen auf die Narrative auswirkten, Anonymität und Intimität möglich sei, welche Gruppen systematisch ausgeschlossen würden und welche Rolle datenschutzrechtliche Aspekte spielten.
Bei allen Möglichkeiten, die der digitale Raum bietet, bleiben jedoch einige $\mathrm{He}$ rausforderungen und Risiken bestehen. - Eine angenehme Atmosphäre oder das Netzwerken kann im Digitalen nur begrenzt erreicht werden, auch die Wahrnehmung mit allen Sinnen ist für Forschende und Interviewteilnehmende nicht gegeben. Ein wichtiger Aspekt ist außerdem die Verstärkung von bestehenden Ungleichheiten, bedingt durch unterschiedliche Zugangs- und Erreichbarkeitsvoraussetzungen, die mit den Kontaktbeschränkungen und Hygienevoraussetzungen im Rahmen der Pandemie im besonderen Maße einhergehen.

\section{Günstige Publikationsbedingun- gen für die Wissenschaft}

Henning Silber, Leiter des Survey Operations Teams, GESIS - Leibniz Institute for the Social Sciences, beleuchtete die Herausforderung der Planung, Umsetzung und Evaluation quantitativer Forschungsdesigns unter Pandemiebedingungen aus Sicht der Sozialforschung. Für kleinere Projekte, die häufig keine Zufallsauswahl vorsehen, existiere heutzutage eine gröBere Flexibilität in der Anpassung des Forschungsdesigns, insbesondere im Hinblick auf Online-Befragungen. Er führte die Rekrutierung über Social-Media-Kanäle an und die im Rahmen der Pandemie ver- stärkte Möglichkeit, entsprechende Drittmittelanträge zu stellen oder in Special Issues zu publizieren.

Um Veränderungen in Bezug auf Einstellungen und Verhaltensweisen vor, während und nach der COVID-19-Pandemie beobachten zu können, sei die Erhebung von Längsschnittdaten als besonderer Datenbedarf bedeutend. Auch die Kombination von verschiedenen Methoden hob Silber für die multiperspektivische Beleuchtung sozialer Tatbestände hervor.

Die Seminar-Abschlussdiskussion ergab, dass der Umgang mit Planungsunsicherheit eine hohe Flexibilität - sowohl im Rahmen qualitativer als auch quantitativer Forschung-erfordert und dass auch in der Pandemie Chancen für die Wissenschaft liegen, sich weiterzuentwickeln. Zudem wurde die Bedeutung des Erfahrungsund Wissensaustausches zwischen Forschern betont - wir lernen alle am besten gemeinsam!

Kontakt:

Das Deutsche Netzwerk Gesundheitskompetenz im Internet: dngk.de Weiterführende Informationen zum Thema: https://dngk.de/webseminar-forschungmaerz-2021/

Kontakt zur Arbeitsgemeinschaft Nachwuchsförderung: agnachwuchs@dngk.de 